\title{
Comparing seed dispersal effectiveness by frugivores at the community level
}

\author{
Aarón González-Castro, ${ }^{1,3}$ María Calviño-Cancela, ${ }^{2}$ and Manuel Nogales ${ }^{1}$ \\ ${ }^{1}$ Island Ecology and Evolution Research Group (CSIC-IPNA), Calle Astrofísico Francisco Sánchez 3, E38206 La Laguna, Tenerife, \\ Canary Islands, Spain \\ ${ }^{2}$ Department of Ecology and Animal Biology, University of Vigo, Faculty of Biology, Campus Lagoas Marcosende, \\ E36310 Vigo, Spain
}

\begin{abstract}
Seed dispersal effectiveness (SDE) is the contribution of dispersers to plant recruitment and is estimated as the product of the number of seeds dispersed (quantity) and the probability of recruitment of each dispersed seed (quality). Although SDE is a key concept in seed dispersal ecology, few studies estimate SDE and none has a community approach. Oceanic islands, with simple communities, are ideal for this purpose. In this study, we compared the SDE of the main types of dispersers (lizards and passerine birds) at the community level in a given habitat. We estimated SDE using a stochastic simulation model parameterized with empirical data on quantity and quality components measured throughout the recruitment process. Although lizards are highly frugivorous and their density was $\sim 20$ times higher than that of birds, lizards and birds dispersed a similar quantity of seeds. This may be due to lower intake of seeds by lizards due to their slower metabolism $(\sim 20$ times lower than birds). This low metabolic rate limits the importance of lizards as seed dispersers, but it is compensated by extraordinarily high lizard densities in the study area ( $\sim 9600$ individuals $/ \mathrm{km}^{2}$ ). High densities of lizards are typical of islands, and this helps to explain why dispersal by lizards seems mainly an island phenomenon. Birds and lizards showed functional complementarity, especially regarding seed dispersal distribution patterns. In fact, lizards dispersed more seeds in shrublands and open sites, and birds in woodlands and beneath canopies, with their joint contribution helping to maximize recruitment. Lizards provided higher SDE than birds for 7 out of 11 plant species. The disperser with a higher quantity for a given plant generally had the higher quality, and plants could be classified as bird- or lizarddependent for dispersal. This dependence increased when considering SDE instead of dispersal quantity only. Moreover, quality was a better predictor of SDE than quantity, which should be considered when parameterizing interaction networks, as this might affect inferences about their architecture.
\end{abstract}

Key words: functional redundancy; germination; island ecology; ornithochory; saurochory; seed rain; seedling recruitment.

\section{INTRODUCTION}

Seed dispersal is a crucial process in the life cycle of plants. It allows seeds to move away from parent plants, avoiding competition and reducing exposure to predators and parasites (Janzen 1970, Connell 1971). It also favors colonization of new areas, enhancing range expansion, metapopulation dynamics, and gene flow (e.g., Nathan 2006).

Internal dispersal of fleshy-fruited plants by frugivorous vertebrates is one of the most studied types of seed dispersal. Frugivores and fleshy-fruited plants usually interact in complex mutualistic networks where an animal often consumes the fruits of several plant species,

Manuscript received 4 April 2014; revised 4 August 2014; accepted 14 August 2014. Corresponding Editor: L. Zanette.

3 Present address: Instituto de Ciencia, Innovación Tecnología y Saberes, Universidad Nacional de Chimborazo, Campus Edison Riera, Salida a Guano, 060150 Riobamba, Ecuador.

E-mail: aarongonzalezcastro@gmail.com while each plant uses the dispersal services of several animal species. Owing to the diffuse nature of these interactions, a community approach to their study is especially appropriate, as it allows a more integrative ecological and evolutionary perspective. This community focus provides better insight into ecosystem functioning and the importance of animal species or groups in maintaining plant biodiversity (Bascompte and Jordano 2007).

Although seed dispersal systems are usually generalized (i.e., seeds are dispersed by a variety of disperser species or groups), dispersal on islands seems to have higher specialization than on mainlands (GonzálezCastro et al. 2012). When various species (or species groups) provide dispersal services to a plant, dispersers can be redundant, providing similar dispersal services and thus being replaceable without loss in plant recruitment, or complementary, providing specific dispersal services, thus having an additive effect on plant recruitment (Zamora 2000, Loiselle et al. 2007). The 
distinction between specialized vs. generalized systems on the one hand, and redundant vs. complementary systems on the other, has important implications for the conservation of biodiversity. The extinction or severe decline of a particular disperser is expected to have less impact in generalized systems that include many disperser species, and when dispersers are redundant, since it can be substituted by its functional equivalents (Zamora 2000).

Quantifying the benefit that plants obtain from dispersers is central to understanding plant-frugivore mutualistic interactions. Seed dispersal effectiveness (hereafter SDE) is the best parameter to estimate this benefit and is a crucial concept in seed dispersal ecology. SDE measures the contribution that dispersers make to plant recruitment and can be estimated as the product of the quantity and quality of seed dispersal (Schupp et al. 2010). Quantity of dispersal refers to the number of seeds dispersed, which is a function of the abundance of dispersers and their feeding preferences (Schupp et al. 2010). Quality of dispersal refers to recruitment probability of dispersed seeds (Schupp et al. 2010). It will depend on the treatment of seeds in the digestive tract of dispersers, and on the seed deposition patterns among sites with different suitability for recruitment. Therefore, quality of dispersal results from interactions between frugivore physiology, behavior, and habitat or landscape features (Côrtes and Uriarte 2013). Few studies on seed dispersal provide comprehensive estimates of SDE (e.g., Godínez-Álvarez et al. 2002, Calviño-Cancela and Martín-Herrero 2009), and none, to our knowledge, have applied a community approach, focusing on a representative sample of all the fleshy-fruited plants and frugivores in a given ecosystem. This is perhaps due to the complexity of measuring SDE, especially at the community level, where one would need to consider each plant-disperser interaction. Such integrative studies become more manageable in relatively simple ecosystems such as oceanic islands (González-Castro et al. 2012).

In the Canary Islands, lizards and birds are the main frugivores. Seed dispersal by lizards (saurochory) has been suggested to be chiefly an island phenomenon (Olesen and Valido 2003), but the relative importance of lizards compared to other sympatric dispersers is still mostly unknown. In this study, we compare the relative importance of lizards and frugivorous passerines as seed dispersers in one of the most threatened ecosystems in the Canary Islands, the thermophilous vegetation. We apply a novel community level approach to focus on SDE provided by both dispersers. We analyze whether birds and lizards consistently provide similar dispersal quantity and quality to plant species and estimate the relative importance of these two components in determining the SDE of these dispersers. We evaluate whether plant dispersal systems are specialized in birds or lizards or otherwise generalized, and whether dispersers are functionally redundant or complementa- ry. In a changing world, populations may decline at an alarmingly rapid pace (Sekercioglu et al. 2004, Tylianakis et al. 2008), especially on oceanic islands (Pimm et al. 1995). A better understanding of the importance of dispersers for plant communities therefore becomes a priority, in order to preserve these mutualistic interactions and maintain biodiversity.

\section{Material and Methods \\ Study site}

Fieldwork was carried out between 2006 and 2011 in one of the best-conserved thermophilous vegetation remnants, situated in the northwest of Tenerife Island, on a steep slope facing north $(\approx 4$-ha patch; Canary Islands, UTM 28R 317523 E, 3138253 N, $220 \mathrm{~m}$ above sea level). The thermophilous belt is the most threatened habitat in the Macaronesian Islands and is located between the coastal shrubland and the laurel forest that usually occupies higher altitudes in the Canary Islands (Fernández-Palacios et al. 2008). It is a heterogeneous formation with shrubland and woodland areas, and its flora is closely related to that of the Mediterranean Basin. The climate is Mediterranean, with mean annual rainfall of 250-450 $\mathrm{mm}$ and mean temperature of 15 $19^{\circ} \mathrm{C}$ (Fernández-Palacios et al. 2008).

The upper part of the slope where the study site is located is dominated by shrubland whereas the woodland predominates in the lower part, toward the Northeast. In each of these two habitats, we distinguished two types of microhabitats: beneath shrubs and trees (hereafter, beneath canopies; with small trees less than $2 \mathrm{~m}$ height classified as shrubs) and open interspaces (clearings with no vegetation or with herbaceous cover only). This results in four types of sites: (1) beneath canopies in shrubland, (2) open areas in shrubland, (3) beneath canopies in woodland, and (4) open areas in woodland, which occupy 55\%, 14\%, 28\%, and $3 \%$ of the study area, respectively. To measure these coverage percentages, we established two sets of transects, one in the upper zone of the slope and the other in the lower zone, in order to get a representative estimation of the different types of habitats and microhabitats in the study area. Each set had $25(10 \mathrm{~m}$ long and $1 \mathrm{~m}$ wide) parallel transects running downhill and spaced $10 \mathrm{~m}$ apart (in an east to west direction). In each transect, we measured the meters covered by each type of site and divided by transect length.

We focused our study on 11 fleshy-fruited plant species (referred to only by genus name hereafter), which account for $99.9 \%$ of fleshy fruits produced in the study area (Appendix A: Table A1). All were Canarian or Macaronesian endemics and were evenly distributed in the study area except for Heberdenia excelsa, a tree especially abundant in the woodland, where soil humidity is higher. Fruit production was monitored in $5-\mathrm{m}^{2}$ plots $(n=20)$ established every $25 \mathrm{~m}$ along a $500-\mathrm{m}$ transect running across the study area in a northeastsouthwest direction. Ripe fruits from all plant species 


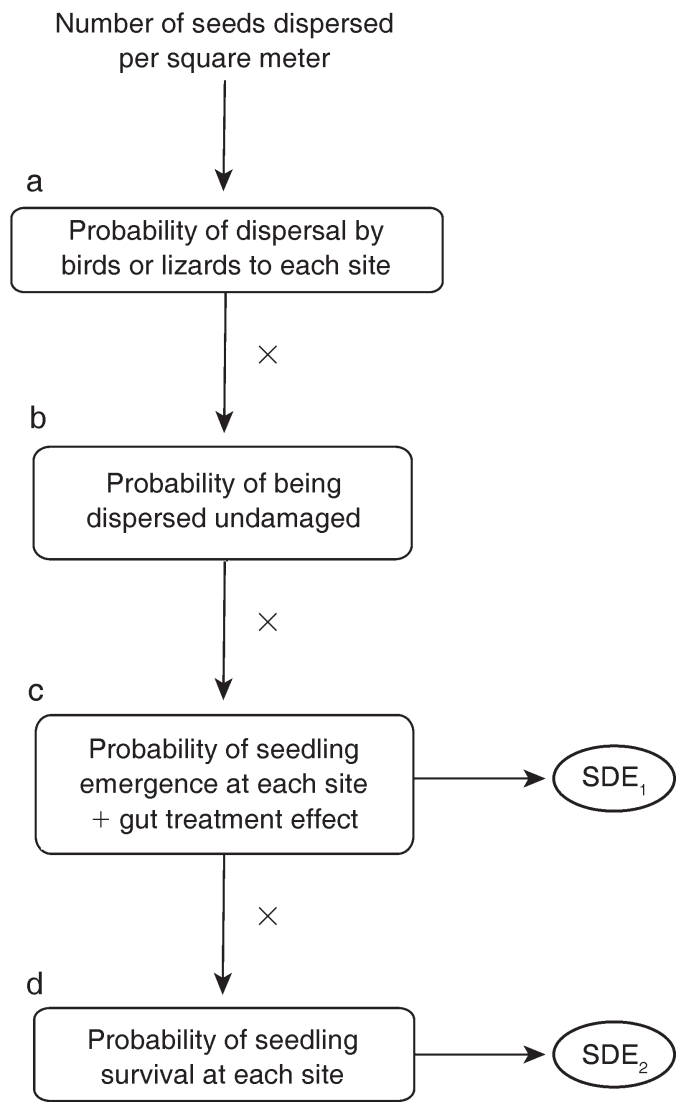

FIG. 1. Structure of the stochastic model to estimate seed dispersal effectiveness (SDE) for species dispersed by passerine birds and lizards (Gallotia galloti) in the study area. The model starts with the number of seeds dispersed per square meter. Transition probabilities applied at each stage of recruitment are shown in boxes, with the symbol " $X$ " referring to the product of these probabilities through the process to estimate the final $\mathrm{SDE}$. $\mathrm{SDE}_{1}$ refers to emergent seedlings and $\mathrm{SDE}_{2}$ to surviving seedlings after one year.

present in the plots were counted every month during 2009.

The assemblage of native seed dispersers is composed of the endemic lizard Gallotia galloti and passerine bird species (all resident species, a subset of those inhabiting the Mediterranean Basin: Sylvia atricapilla, S. melanocephala (see Plate 1), Turdus merula, and Erithacus rubecula, with Cyanistes teneriffae as mostly a fruit predator). Estimated densities averaged 9570 and 520 individuals $/ \mathrm{km}^{2}$ for lizards and birds, respectively, in the study area. Densities were estimated twice a month for one year (from March 2010 to February 2011) with a $500-\mathrm{m}$ transect (the same used to sample fruit production). A band $4 \mathrm{~m}$ wide for lizards and a band $25 \mathrm{~m}$ wide for birds were surveyed, where all individuals (seen or heard) were counted. The small size of the bands we used maximizes detectability of animals and also makes easy to determine whether observed animals are within or outside the band. Other present day endangered native species, such as Columba junoniae, Corvus corax, and Canarian giant lizards (Gallotia spp.; see Plate 1), might have been important seed dispersers in the past (Fernández-Palacios et al. 2008), but their current role in the thermophilous vegetation is probably negligible on most islands. Regarding nonnative dispersers, only some feces of rabbits (Oryctolagus cuniculus) were observed to contain seeds in the study area, but seeds removed from them did not germinate (data not shown).

\section{Estimation of the SDE provided by frugivores}

Simulation model description.-SDE was estimated as the density of seedlings of each plant species contributed by either birds or lizards in the study area. For this, we used a stochastic simulation model (Fig. 1) that follows the seedling recruitment processes and was parameterized with quantity and quality components of SDE measured in the field and greenhouse (see Simulation model parameterization). The model consists of a series of stages connected by processes, each with its own empirically measured set of transition probabilities. It starts by distributing the seeds into the four site types (each combination of habitat and microhabitat) according to the densities of seeds sampled in the field. Then, it applies the transition probabilities corresponding to (1) dispersal by either birds or lizards (percentage of seeds in bird or lizard defecations/regurgitations in the sampling plots), (2) seed damage caused through ingestion by animals (percentage of damaged seeds in bird and lizard feces/regurgitations), (3) seedling emergence, considering both the effect of the site (percentage of control seeds, taken from the mother plants, that germinate in each site type) and the effect of seed ingestion by dispersers (ratio between the probability of seedling emergence obtained from seeds defecated/ regurgitated by birds or lizards and that of control seeds) and, last, (4) seedling survival (percentage of seedlings surviving after 1 year). The original sample of transition probabilities for each process, measured empirically, was resampled 1000 times by random selection with replacement (bootstrapping; Manly 1998). The final output of each simulation iteration (either the seedlings emerged $\left[\mathrm{SDE}_{1}\right]$ or seedlings that survived to year one $\left[\mathrm{SDE}_{2}\right]$ ) is the result of the product of the randomly selected transition probabilities at each stage. To compute SDE for birds and lizards, the estimated number of emerged seedlings $\left(\mathrm{SDE}_{1}\right)$ or those surviving to year one $\left(\mathrm{SDE}_{2}\right)$ in each site type was subsequently weighted by the relative cover of those site types in the study area.

The stochastic model allows us to track the fate of individuals (seeds or seedlings) throughout the process. Thus, we can quantify the individuals reaching every recruitment stage, and estimate the quantity and quality components for each disperser and plant species. For each simulation iteration, we computed the SDE provided by dispersers $\left(\mathrm{SDE}_{1}\right.$ and $\left.\mathrm{SDE}_{2}\right)$, the quantity (total number of seeds dispersed by birds or lizards), and 
quality (SDE/quantity), and plotted the average SDE (of a total of 1000 iterations) for each disperser-plant pair in a landscape graph using the quantity and quality components.

Simulation model parameterization.-The parameters used in the model are shown in Table A2, and were obtained using the following methodology.

1. Amount of seeds dispersed per site and disperser.To estimate the quantity of seed dispersal (Fig. 1a) for each plant species, we monitored 80 seed traps (20 traps per each combination of habitat and microhabitat) every month (from March 2010 to February 2011). Seed traps consisted of $1-\mathrm{m}^{2}$ square trays $2 \mathrm{~cm}$ deep and made out of green plastic mesh ( $1 \mathrm{~mm}$ aperture), to retain feces and provide good drainage. Traps were placed parallel to a path (15 m away to avoid edge effects) running across the woodland and shrubland areas. The distance from the starting point of the path as well as between seed traps was established by generating random numbers between 5 and 20 (i.e., 5-20 m distances). Feces of passerine birds and lizards and regurgitations of the former were easily distinguishable in the traps. Seeds contained in feces and regurgitations were identified and counted.

2. Seed damage caused by passage through the digestive tract of animals.-We used a dissecting stereomicroscope $(16 \times)$ to visually determine the percentage of damaged seeds (Fig. 1b) in bird and lizard feces and regurgitations $(n=624$ and 621 regurgitations and feces for birds and lizards, respectively) collected at the study site from 2006 to 2009 (total: 5279 seeds). Bird feces and regurgitations were collected during mistnetting sessions and at water feeders (removed before sampling seed rain and disperser densities). Lizard feces were collected along a $500 \times 2 \mathrm{~m}$ transect (the same used to estimate lizard and bird densities).

3. Seedling emergence.-We estimated both the effect of site and ingestion by dispersers on seedling emergence (Fig. 1c). To test the site effect we sowed control seeds ( $\sim 2 \mathrm{~cm}$ apart) of the 11 plant species in $101-\mathrm{m}^{2}$ square plots per site type (each habitatmicrohabitat combination, 40 plots in total). Plots were distributed in the study area as described for seed traps. Control seeds were obtained from depulped ripe fruits picked from those available on randomly selected plants ( $n=15-23$ mother plants). We sowed 10436 seeds in total, with an average 261 seeds per plot and 23 seeds per plant species. The number of seeds per species per plot varied between 9 and 67 seeds, being constrained by fruit availability. We installed a metal mesh covering the plots to avoid potential disturbances. Seeds were sown in October 2010 and seedling emergence was surveyed every 15 days until the end of March 2011, thus comprising the rainy season in the Canaries, when seedling emergence mainly occurs (Rodríguez et al. 2007).

The effect of ingestion by dispersers was computed as the ratio between the probability of seedling emergence from undamaged seeds defecated/regurgitated by birds or lizards and the probability of seedling emergence from control seeds. This ratio equals 1 when there was no effect (emergence of control seeds equals that of defecated seeds), is lower than 1 when seed ingestion has a detrimental effect, and higher than 1 when positive. Control and ingested seeds were obtained as described previously. Seeds collected in each fruiting season (2006-2007, 20072008, and 2008-2009) were sown the following October. Seeds from each treatment (1698 for control, 1316 for bird, and 1526 for lizard; 4540 seeds in total, with an average of 151 seeds per treatment per species) were individually sown in $4-\mathrm{cm}^{2}$ pots filled with a standard substrate $(50 \%$ peat and $50 \%$ agricultural soil), and were distributed randomly in the greenhouse. Pots were watered every two days and seedling emergence was monitored every five days for six months, until the end of March.

The probability of seedling emergence for seeds defecated by birds and lizards in each site type was estimated as the product of the probability for control seeds in each site and the effect of seed ingestion, computed as previously explained.

4. Seedling survival.-To test the effect of site on seedling survival (Fig. 1d) we established $80\left(2-\mathrm{m}^{2}\right)$ plots within each combination of habitat and microhabitat (20 per site type), distributed as described above for seed traps. This was in March 2010 just before the dry season spanning from April to September, the most severe for seedling establishment and survival. Naturally emerging seedlings of all study species were then counted and marked. Survival of marked seedlings was then recorded until September 2010 (1-yr-old seedlings). Seedling survival could not be estimated for Canarina, Scilla, and Tamus, as they are geophytes that pass the dry season underground and, after that, it was difficult to distinguish between newly emerged seedlings and those surviving the dry season.

\section{Statistical analyses}

We did two sets of analyses, one with empirical data and the other with the output from the simulation model. We used the empirical data to (1) compare the quantity of seeds dispersed per square meter by birds and lizards, and their distribution among sites (quasigeneralized linear model with a Poisson error distribution) and (2) to test the effects of dispersers (plus control in the case of seedling emergence) and plant species on seed damage and seedling emergence, as well as the effect of habitat, microhabitat and plant species on seedling emergence and survival (GLM with binomial error distribution). All interactions among factors were included in the GLMs. G tests (Sokal and Rohlf 1995) were used to assess the effect of dispersers (or dispersers plus control) on seed damage and seedling emergence, and the effect of site (each combination of habitats and microhabitats) on seedling emergence and survival for 

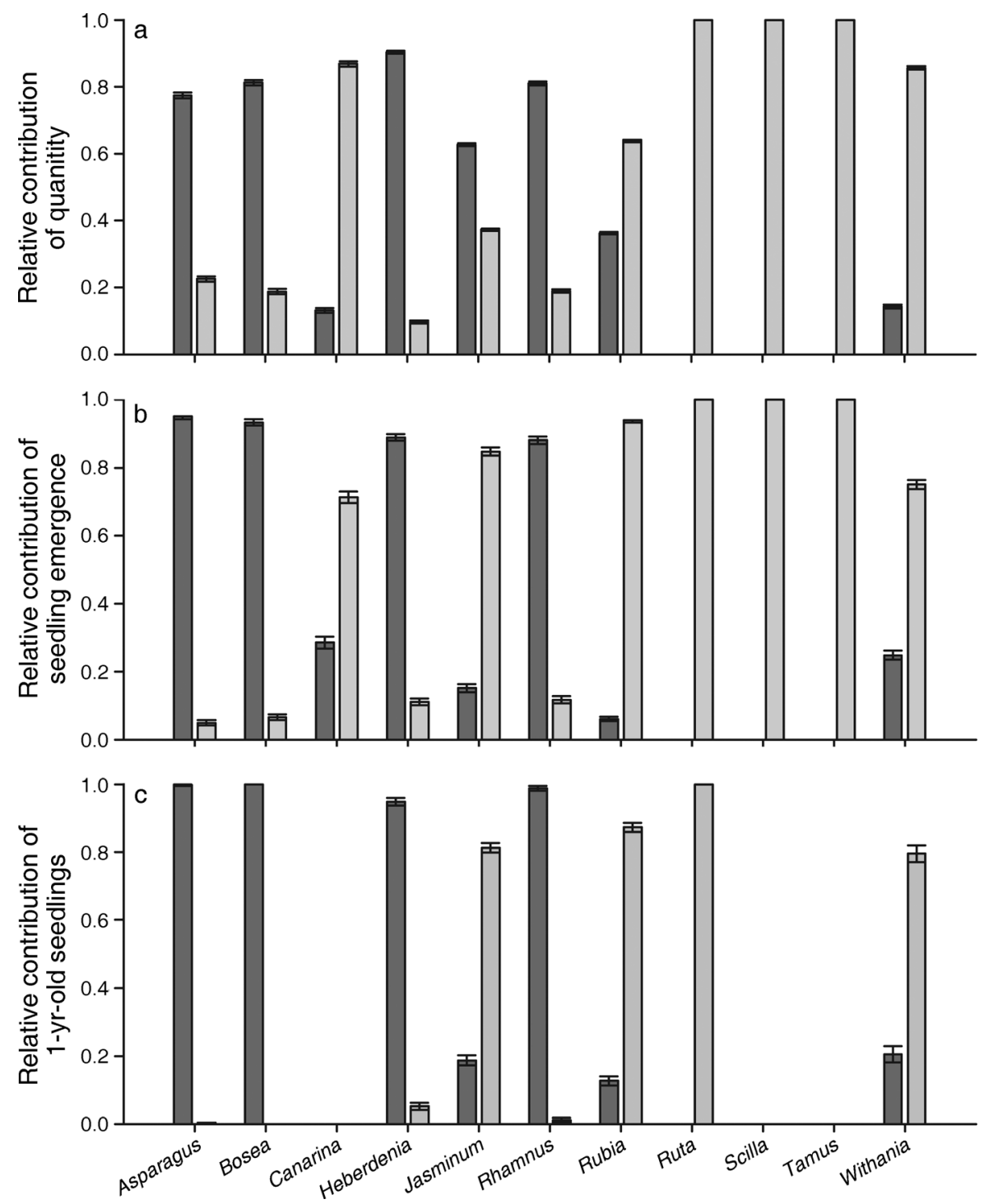

FIG. 2. Relative contribution of birds (dark gray bars) and lizards (light gray bars) to (a) the quantity component (number of seeds dispersed), (b) number of seedlings emerged $\left(\mathrm{SDE}_{1}\right)$, and (c) number of surviving seedlings after one year ( $\left.\mathrm{SDE}_{2}\right)$, for each plant species studied. No seedling survival could be estimated for Canarina, Tamus, and Scilla. Values are proportions (mean \pm $\mathrm{SE})$.

each plant species separately (results shown in Appendix B: Figs. B1, B2, B4, and B5). The Bonferroni correction was used for pairwise comparisons of treatments in all cases.

With the model outputs, we tested the significance of the effect of disperser and plant species (as factors) and the effect of quantity and quality components (as covariables) on the $\mathrm{SDE}_{1}$ and $\mathrm{SDE}_{2}$, by using an ANCOVA. We computed Pearson product-moment correlations between the quantity and quality components of SDE and used a linear regression analysis to estimate, for each combination of plant species and disperser, the percentage of variance of SDE explained by the quantity and quality components (as the coefficient of determination, $R^{2}$ ).

\section{RESULTS}

Quantity of seed dispersal provided by birds and lizards

Our empirical data indicated that the quantity of seeds dispersed by birds and lizards were similar when all plant species were pooled together $\left(20.33\right.$ seeds $/ \mathrm{m}^{2}$ vs. 26.73 seeds $/ \mathrm{m}^{2}$ dispersed by birds and lizards, respectively; $F_{4,75}=2.48, P=0.117$ ). However, for each plant species separately, there was a predominance of either lizards or birds as dispersers $(>60 \%$ of seeds dispersed by the most important disperser in all cases, and $\geq 75 \%$ in 9 out of the 11 plant species studied; Fig. 2a). Lizards were predominant for six plant species (the sole disperser for three of them), and birds for the other five (Fig. 2a). 
TABLE 1. Results of ANCOVA to test for the effect of type of disperser, plant species, and seed dispersal effectiveness (SDE) components (quantity and quality) on $\mathrm{SDE}_{1}$ and $\mathrm{SDE}_{2}$.

\begin{tabular}{|c|c|c|c|c|c|c|}
\hline \multirow[b]{2}{*}{ Explanatory variables } & \multicolumn{3}{|c|}{$\mathrm{SDE}_{1}$} & \multicolumn{3}{|c|}{$\mathrm{SDE}_{2}$} \\
\hline & $F$ & $\mathrm{df}$ & $P$ & $F$ & df & $P$ \\
\hline Disperser & 1986.84 & 1 & $<0.001$ & 1773.90 & 1 & $<0.001$ \\
\hline Plant species & 960.91 & 10 & $<0.001$ & 1384.58 & 7 & $<0.001$ \\
\hline Quantity component & 12340.52 & 1 & $<0.001$ & 6558.74 & 1 & $<0.001$ \\
\hline Quality component & 19395.92 & 1 & $<0.001$ & 35547.9 & 1 & $<0.001$ \\
\hline Disperser : plant species & 280.478 & 7 & $<0.001$ & 291.59 & 6 & $<0.001$ \\
\hline
\end{tabular}

Note: $\mathrm{SDE}_{1}$ refers to emergent seedlings and $\mathrm{SDE}_{2}$ to surviving seedlings after one year.

\section{Quality of seed dispersal provided by birds and lizards}

The probability of seeds surviving through the different stages of recruitment (quality of dispersal, as measured in the field and greenhouse) differed according to the disperser and the plant species. Different patterns were identifiable, depending on the stage considered.

Effects of dispersers' digestive tract on seed damage and seedling emergence.-In general, ingestion by birds caused less damage to seeds than by lizards $\left(\chi_{1}^{2}=\right.$ 13.11, $P<0.001$; Appendix B: Fig. B1). Seed damage differed also among plant species $\left(\chi_{7}^{2}=133.22, P<\right.$ $0.001)$ but there was no significant interaction between disperser and plant species $\left(\chi_{7}^{2}=11.095, P=0.135\right)$. Regarding seedling emergence, there was a significant effect of treatment (passage through birds, lizards, or control; $\left.\chi_{2}^{2}=241.36, P<0.001\right)$, plant species $\left(\chi_{10}^{2}=\right.$ 178.98, $P<0.001)$, and the interaction between these two factors $\left(\chi_{17}^{2}=274.57, P<0.001\right.$; Fig. B2). This indicated that seed ingestion affected plant species differently, having a significant effect in 8 of the 11 species $(73 \%$, detrimental in all cases; Fig. B2). This effect was more detrimental for seeds ingested by birds than by lizards (six out of eight species; Fig. B2).

Seed dispersal among sites and site suitability for seedling emergence and survival.-Birds and lizards showed contrasting patterns of seed dispersal at the habitat and microhabitat levels; with significant interactions between disperser and habitat $\left(F_{4,75}=23.925, P\right.$ $<0.001)$ and between disperser and microhabitat $\left(F_{4,75}\right.$ $=218.755, P<0.001)$. Birds deposited more seeds in woodland than in shrubland, and more beneath canopies than in open areas, whereas lizards showed the opposite pattern (Appendix B: Fig. B3).

Site suitability for seedling emergence and survival differed among plant species; there were significant second- and third-order interactions between habitat, microhabitat, and plant species (Tables B1 and B2). Seedling emergence tended to be higher beneath shrubs than in the open in shrublands but similar between microhabitats in woodlands (see "All species" in Fig. B4), and seedling survival tended to be higher beneath canopies than in the open (Ruta and Withania were the only exceptions; Fig. B5).

\section{SDE provided by birds and lizards}

$\mathrm{SDE}_{1}$ and $\mathrm{SDE}_{2}$ were significantly affected by the type of disperser, the plant species and by the quantity and quality components $(P<0.001$ in all cases; see Table 1$)$. For the whole community, lizards provided higher SDE than birds, both for $\mathrm{SDE}_{1}(66.2 \%$ vs. $33.8 \%)$, and $\mathrm{SDE}_{2}$ (71.4\% vs. $28.6 \%$ ). However, the relative importance of lizards and birds differed according to the plant species (Fig. 2b and c; $P<0.001$; Table 1). In all cases, the relative contribution of the disperser with the highest SDE was $\geq 70 \%$, being higher than $90 \%$ in 6 out of 11 plant species when considering $\mathrm{SDE}_{1}$ and 5 out of 8 when considering $\mathrm{SDE}_{2}$ (Fig. 2). Lizards were the main contributors to seedling emergence for 7 out of 11 plants (c. $65 \%$ ).

The highest SDE values were reached by lizard interactions with Rubia and Canarina for $\mathrm{SDE}_{1}$ and with Rubia for $\mathrm{SDE}_{2}$, with relatively high quantity and quality values (Fig. 3). The rest of interactions reached comparatively low SDE values. In bird-plant interactions, this was mainly due to negatively correlated quantity and quality components $(r=-0.863, P=$ $0.006, n=8$, for $\mathrm{SDE}_{1} ; r=-0.482, P=0.273, n=7$, for $\mathrm{SDE}_{2}$; Pearson product-moment correlations), with SDE values lying along the $x$ - or $y$-axes (Fig. 3). For lizards, quantity and quality were not negatively correlated $(r=0.108, P=0.752, n=11$, and $r=$ $0.388, P=0.342, n=8$ ) and low SDE values were mostly due to low values of both quantity and quality (Fig. 3).

\section{Quantity and quality as determinants of SDE}

The effect of dispersal quantity and quality on SDE varied depending on the disperser and the plant species, but quality was more important than quantity in determining SDE in all but one case (Canarina when dispersed by birds; Appendix C: Table C1). On average, quantity explained $11 \%$ and $3 \%$ of the variance in $\mathrm{SDE}_{1}$ and $\mathrm{SDE}_{2}$, respectively, whereas quality explained $63 \%$ and $76 \%$ (Table C1). There were wider differences between dispersers in quality across plants (1.08 to 60 times with $\mathrm{SDE}_{1}$, and 1.13 to $\sim 1300$ times with $\mathrm{SDE}_{2}$ ) than in quantity (2-14 times; Fig. 3). For any given plant, the disperser with the highest quantity provided the highest quality, in all cases except Jasminum. 

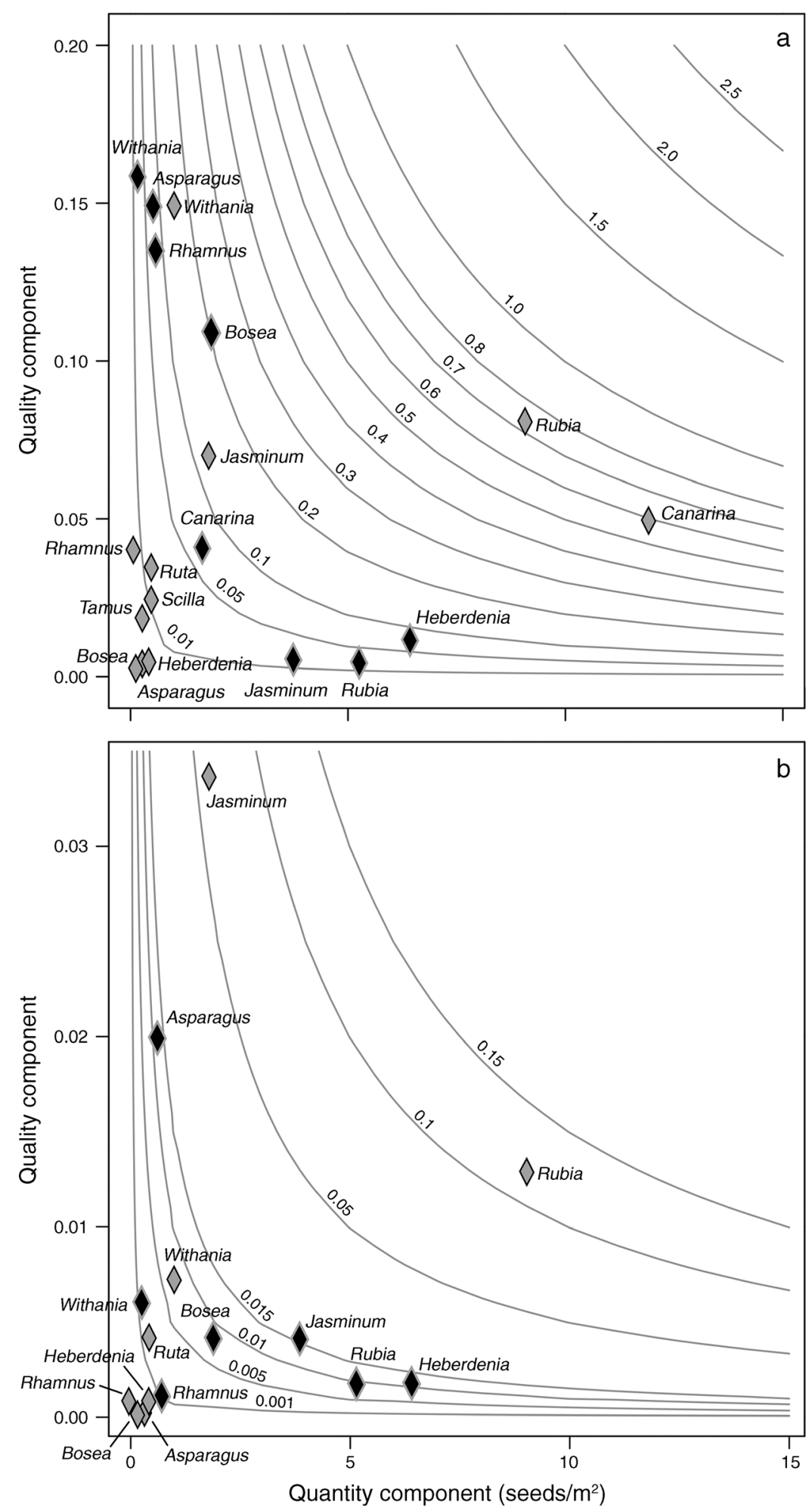

FIG. 3. Seed dispersal effectiveness (SDE) landscapes showing the distribution of the estimated values of (a) SDE $_{1}$ (no. seedlings emerged $/ \mathrm{m}^{2}$ ) and (b) $\mathrm{SDE}_{2}$ (no. 1-yr-old seedlings $/ \mathrm{m}^{2}$ ) provided by birds (black diamonds) and lizards (gray diamonds) for each plant species. Isoclines join all combinations of quantity and quality values giving the same SDE values (numbers on the isoclines). The quantity component refers to the estimated number of seeds dispersed per square meter, and the quality to the probability of each seed becoming an emergent seedling or a 1-yr-old seedling, as estimated with the stochastic model. 


\section{Discussion}

Our results show the functional complementarity between birds and lizards in the thermophilous vegetation of the Canary Islands. The use of SDE landscapes provided us with a visual and very intuitive assessment of this, and the relationship between quantity and quality components across the 19 plant-disperser interactions studied. Both birds and lizards dispersed most of the fleshy-fruited plants. However, about $30 \%$ of plant species (3 out of the 11 studied) were dispersed solely by lizards and none were dispersed solely by birds. This contrasts with Mediterranean shrublands and woodlands on the mainland (e.g., the Mediterranean basin), where passerine birds are the main seed dispersers (Herrera 1995). Moreover, lizards provided higher SDE (pooling all plant species) and were the main contributors to seedling emergence for 7 out of 11 plants, which suggests a higher dependence on dispersal by lizards than by birds in this ecosystem. This might be related to the more ancient presence of Gallotia lizards in the Canary Islands, which might have favored their greater integration into interaction networks compared to passerines, since this integrations takes time (Brändle et al. 2008). Gallotia began colonizing the Canary Islands shortly after they were formed (17-20 million years, Cox et al. 2010), in contrast to the recent colonization by frugivorous passerines. The Canaries were colonized by avifauna mainly in the Pleistocene, 0.01 to 2.3 million years ago, and $\sim 4-13$ thousand years ago by the current frugivorous passerines (Illera et al. 2012).

\section{SDE landscapes}

The SDE landscapes showed very disparate positions of the SDE values of lizards and birds for all plant species, which reveals their low functional equivalence. Moreover, for each plant species separately, there was a clear predominance of either lizards or birds as dispersers. High SDE values of one disperser were accompanied by low values of the other, so that plants could be regarded as mostly bird dependent or lizard dependent for seed dispersal in this ecosystem.

SDE landscapes also showed a wide variability in quantity and quality components across plant species, so one disperser type was not consistently more effective than the other in either quantity or quality of dispersal. Moreover, although the disperser with higher quantity for a given plant usually provided better dispersal quality than the other, the exact values of quantity and quality were not positively correlated. They showed weak or even negative correlations, especially for birds, and this decoupling between components limited SDE. Variability and decoupling was also observed for the quality subcomponents (e.g., lizards caused higher seed damage than birds but had a less detrimental effect on seedling emergence from undamaged seeds. This is a result of the heterogeneity in the characteristics of the animals, the plants and the environmental conditions that affect the quantity and quality of dispersal, and the interactions among them (reviews in Jordano 2000, Schupp et al. 2010). Moreover, the context dependence of the subcomponents of quantity and quality of dispersal favors shifts in space and time in the SDE landscapes (Schupp et al. 2010). These shifts can be especially drastic for interactions with SDEs along the $x$-axis (quantity) or $y$-axis (quality), for which changes in the limiting component would have a strong impact on SDE. For instance, greater bird abundance raises the quantity of dispersal by birds, increasing SDE in species for which birds provide high dispersal quality (e.g., Asparagus). Thus, a disperser important for one plant may be less important for another, and this pattern of plant dependence on dispersers may vary circumstantially (context dependency; see, e.g., Calviño-Cancela and Martín-Herrero 2009). Such variability reflects great complexity and is an important property of seed dispersal interaction networks, as it enhances their robustness (see Bascompte and Jordano 2007).

$\mathrm{SDE}$ is very complex and time consuming to estimate, which has raised the interest in finding simpler methods (Calviño-Cancela 2011) or valid surrogates (Vázquez et al. 2005) to parameterize complex interaction networks. In this study, dispersal quality was more important than quantity in determining SDE (thus a better surrogate, see also Calviño-Cancela and Martín-Herrero 2009), because it varied more widely than quantity. Moreover, plant dependence on a particular disperser increased when considering $\mathrm{SDE}_{1}$ instead of just quantity, and even more with $\mathrm{SDE}_{2}$, when further measures of dispersal quality were added (seedling survival to year one). This suggests that inferences on the architecture of interaction networks might change substantially when more comprehensive estimates of SDE are incorporated.

\section{Dispersal quantity}

In any system, the quantity of dispersal provided by an animal depends on its local abundance and the amount of seeds processed per individual per unit time. This in turn depends on the amount of total food processed (derived from the animal's metabolic rate) and the predominance of fruits in the diet (degree of frugivory; see also Schupp et al. 2010). In our study area, lizards are more frugivorous than birds (frequency of occurrence of seeds in feces and regurgitations, 98\% for lizards vs. 58\% for birds; see Rodríguez et al. [2008] for data on lizards; A. González-Castro, unpublished data, for passerines), and had $\sim 20$ times higher density. However they dispersed similar quantities of seeds, when pooling all plant species. Dispersal quantity by lizards thus seems to be limited by their low rates of food intake associated with low metabolic rates $(\sim 20$ times lower for reptiles [ectothermic] than for birds [endothermic] of similar sizes and diet; see Nagy et al. 1999). These low metabolic rates of lizards constrain their role as dispersers in ecosystems. As a general rule, for lizards to have an important role as dispersers, their low fruit 

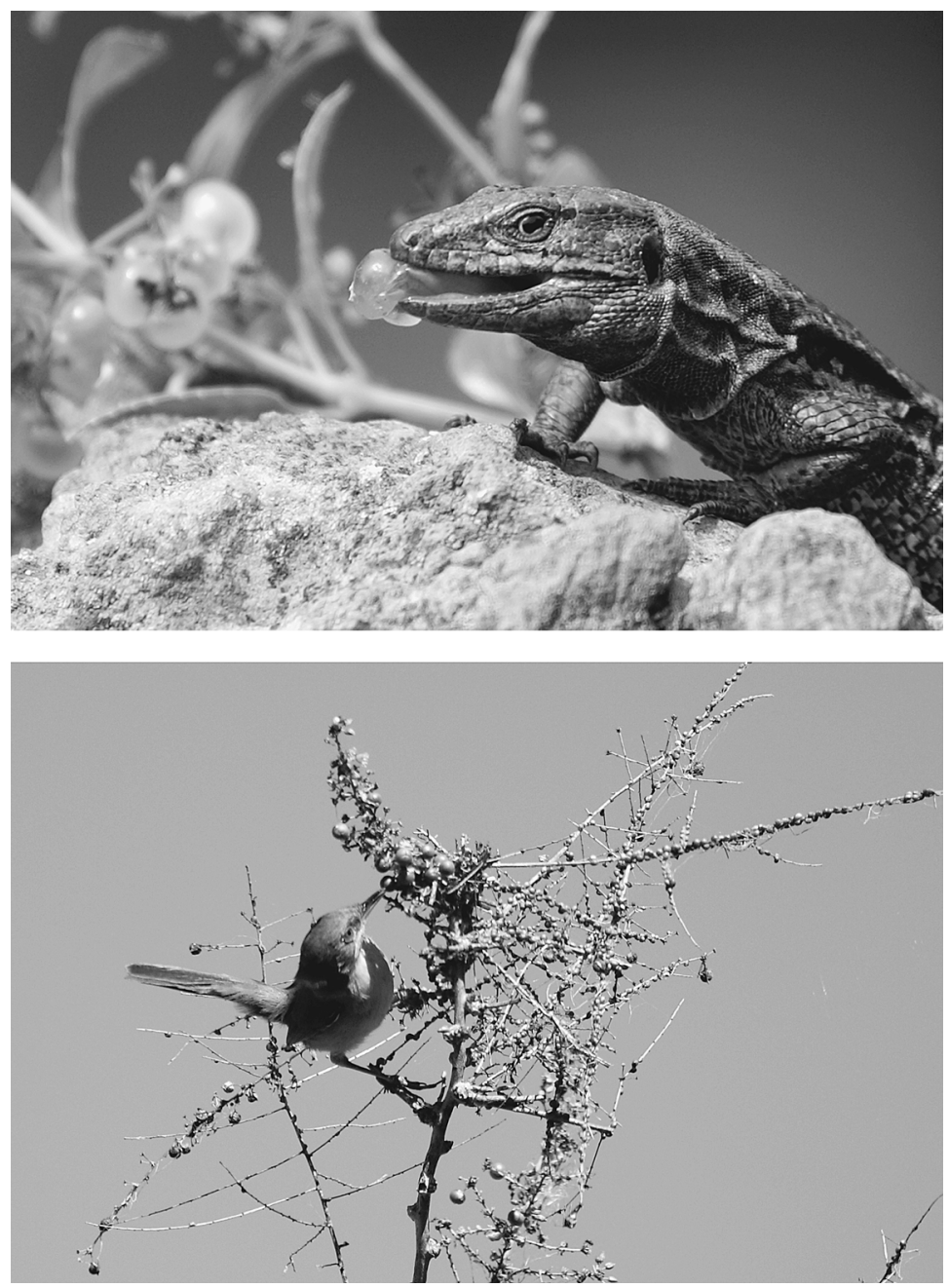

Plate 1. (Top) Canarian endemic lizard (Gallotia galloti) eating Rubia fruticosa fruits and (bottom) Sardinian Warbler (Sylvia melanocephala) eating Asparagus plocamoides fruits. Photo credits: Beneharo Rodriguez.

intake per individual would have to be compensated by high densities because, on average, 20 lizards are needed to equal the role of one bird, of similar size and diet (Nagy et al. 1999). Such compensation (or even overcompensation) is more probable on islands, where lizard densities are recorded as much higher than on the mainland (15 times higher as global average: Buckley and Jetz 2007). This may help to explain why seed dispersal by lizards is considered to be mainly an island phenomenon. Previous studies have attributed it to a higher degree of frugivory by island lizards (Olesen and Valido 2003). However, even a highly frugivorous lizard such as G. galloti would have a minor role as disperser at the usual mainland densities: at 15 times less density, $G$. galloti would disperse $\sim 2$ seeds $/ \mathrm{m}^{2}$ in our study area, all other things held constant, compared to 20 seeds $/ \mathrm{m}^{2}$ by birds. Therefore, the high level of seed dispersal by lizards on islands could be a consequence of the joint effect of both the higher degree of frugivory by lizards and higher lizard density on islands than on mainland.

\section{Dispersal quality}

Birds and lizards differed also in the quality of dispersal they provided, which can be explained by physiological differences (especially thermal) between them. Low metabolic rates of lizards are related to their long gut retention times ( $\sim 7$ days vs. $\sim 0.3-1.5 \mathrm{~h}$ for passerines; Valido 1999, Jordano 2000), which probably caused the higher seed damage than that by birds. Moreover, as ectotherms, lizards spend more time in open interspaces, exposed to the sun (Díaz 1994), in contrast to frugivorous passerines, that spend more time perched on shrubs and trees and prefer areas of denser vegetation (e.g., Debussche et al. 1985, Alcántara et al. 2000). In addition to these physiological differences, lizards have limited movement ranges compared to birds (e.g., Molina-Borja [1985] for data on lizards, Jordano et al. [2007] for data on passerines). This would be expected to affect seed dispersal distances and thus, gene flow, meta- 
population dynamics and migration ability of plant species (e.g., in response to climate change; Nathan 2006).

The effect of site was variable among plants in regard to seedling emergence, but more consistent for seedling survival, with a facilitative effect of vegetation cover, resulting in lower mortality for seeds dispersed by birds. Vegetation cover reduces water loss, usually the most important cause of seedling mortality under Mediterranean climates (Lloret et al. 2005). The complementarity between lizards and birds with respect to seed distribution patterns favored a wider distribution of seedlings among sites and helped to maximize recruitment, which occurred in both open sites and beneath canopies. This complementarity between dispersers could also contribute to model vegetation patterns, with plants dispersed mostly by lizards being the most probable colonizers of open areas. Since the fruits of some of them are also consumed by birds, they could become nuclei for species dispersed mostly by birds, facilitating their establishment (e.g., Debussche et al. 1985). This may favor the mixing of lizard- and bird-dependent plant species observed in the study area.

\section{Implications for ecosystem robustness}

We found recruitment to be predominantly bird or lizard dependent for different plant species. On islands, a higher dependence of plants on a single or a few species may be more frequent than on continents (González-Castro et al. 2012). This may result in higher vulnerability to disturbances, which may lead to mutualism disruptions when local extinctions of dispersers occur (Rodríguez-Pérez and Traveset 2010, Calviño-Cancela et al. 2012). In our ecosystem, plants that have depended mostly on lizards for dispersal (and especially those dispersed solely by them) would be more vulnerable, as they currently rely on a single species after giant lizards became rare in the Canary Islands (Valido 1999), whereas there are five species of bird dispersers. Plants dispersed by both birds and lizards would be expected to be less vulnerable because, if the main disperser becomes extinct, the less important partner might guarantee some recruitment for these plant species. Multispecies or multi-guild dispersal systems are thus expected to be advantageous in the long term. However, owing to the complementarity between lizards and birds, if the main disperser for a plant species becomes extinct, the recruitment level provided by the less important disperser would be reduced if compared to that provided by the main disperser, which would affect the plant's population dynamics.

As far as we know, this study is the first comprehensive attempt to compare the effectiveness of the main groups of seed dispersers in a particular ecosystem at the community level. Comprehensive studies on SDE are still very scarce and more research is needed into different ecosystems to determine how common is such strong dependence on a particular disperser or functional group, and how this dependence changes over broad spatial and temporal scales (context dependence). The degree of dependence on particular species or groups for recruitment has important implications from both evolutionary and ecological perspectives. It is essential in determining the robustness of interaction networks (see Bascompte and Jordano 2007) on which the long-term persistence of interacting species depends, being thus crucial for biodiversity maintenance.

\section{ACKNOWLEDGMENTS}

We are especially grateful to Airam Rodríguez, David Padilla, Beatriz Rumeu, Daniel González, Benito Pérez, Yurena Gavilán, Patricia Marrero, Elsa Bonnaud, and Concepción Nieves, who helped at different stages of the study. Javier Rodríguez-Pérez, Alfredo Valido, and four anonymous referees made useful comments to a previous version of the manuscript. A. González-Castro benefited from a JAE-PRE fellowship from the Spanish National Research Council (CSIC), and M. Calviño-Cancela was supported by an Isidro Parga Pondal contract (Xunta de Galicia). This work was financed by the Spanish Ministry of Science and Education project (CGL2007-61165/BOS), supported by FEDER funds from the European Union. The Cabildo of Tenerife and the landowner (Teobaldo Méndez) gave permission to work at the study site. Tagoro Medioambiente provided its greenhouse to perform the seedling emergence experiments. The Universidade de Vigo gave logistical support to A. González-Castro from February to April 2012. A. González-Castro and M. CalviñoCancela contributed equally to this work.

\section{Literature Cited}

Alcántara, J. M., P. J. Rey, F. Valera, and A. M. SánchezLafuente. 2000. Factors shaping the seedfall pattern of a bird-dispersed plant. Ecology 81:1937-1950.

Bascompte, J., and P. Jordano. 2007. Plant-animal mutualistic networks: the architecture of biodiversity. Annual Review of Ecology, Evolution, and Systematics 38:567-593.

Brändle, M., I. Kühn, S. Klotz, C. Belle, and R. Brandl. 2008. Species richness of herbivores on exotic host plants increases with time since introduction of the host. Diversity and Distributions 14:905-912.

Buckley, L. B., and W. Jetz. 2007. Insularity and the determinants of lizard population density. Ecology Letters 10:481-489.

Calviño-Cancela, M. 2011. Simplifying methods to assess site suitability for plant recruitment. Plant Ecology 212:13751383.

Calviño-Cancela, M., M. Escudero, J. Rodríguez-Pérez, E. Cano, P. Vargas, G. Velo-Antón, and A. Traveset. 2012. The role of seed dispersal, pollination and historical effects on genetic patterns of an insular plant that has lost its only seed disperser. Journal of Biogeography 39:1996-2006.

Calviño-Cancela, M., and J. Martín-Herrero. 2009. Effectiveness of a varied assemblage of seed dispersers of a fleshyfruited plant. Ecology 90:3503-3515.

Connell, J. H. 1971. On the role of natural enemies in preventing competitive exclusion in some marine animals and in rain forest trees. Pages 298-312 in P. J. Den Boer and G. Gradwell, editors. Dynamics of populations. PUDOC, Wageningen, The Netherlands.

Côrtes, M. C., and M. Uriarte. 2013. Integrating frugivory and animal movement: a review of the evidence and implications for scaling seed dispersal. Biological Reviews 88:255-272. 
Cox, S. C., S. Carranza, and R. P. Brown. 2010. Divergence times and colonization of the Canary Islands by Gallotia lizards. Molecular Phylogenetics and Evolution 56:747-757.

Debussche, M., J. Lepart, and J. Molina. 1985. Seed dispersal by birds, effect of vegetation structure and influence on succession in a Mediterranean Region. Acta OecologicaOecologia Generalis 6:65-80.

Díaz, J. A. 1994. Field thermoregulatory behavior in the Western Canarian lizard Gallotia galloti. Journal of Herpetology 28:325-333.

Fernández-Palacios, J. M., R. Otto, J. D. Delgado, J. R. Arévalo, A. Naranjo, F. González Artiles, C. Morici, and R. Barone. 2008. Los bosques termófilos de Canarias. Proyecto LIFE04/NAT/ES/000064. Cabildo Insular de Tenerife, Santa Cruz de Tenerife, España.

Godínez-Álvarez, H., A. Valiente-Banuet, and A. RojasMartínez. 2002. The role of seed dispersers in the population dynamics of the columnar cactus Neobuxbaumia tetetzo. Ecology 83:2617-2629.

González-Castro, A., A. Traveset, and M. Nogales. 2012. Seed dispersal interactions in the Mediterranean Region: contrasting patterns between islands and mainland. Journal of Biogeography 39:1938-1947.

Herrera, C. M. 1995. Plant-vertebrate seed dispersal systems in the Mediterranean - ecological, evolutionary, and historical determinants. Annual Review of Ecology and Systematics 26: 705-727.

Illera, J. C., J. C. Rando, D. S. Richardson, and B. C. Emerson. 2012. Age, origins and extinctions of the avifauna of Macaronesia: a synthesis of phylogenetic and fossil information. Quaternary Science Reviews 50:14-22.

Janzen, D. H. 1970. Herbivores and the number of tree species in tropical forests. American Naturalist 104:501-529.

Jordano, P. 2000. Fruits and frugivores. Pages 125-165 in M. Fenner, editor. Seeds: the ecology of regeneration in plant communities. Second edition. CAB International, Oxford, UK.

Jordano, P., C. García, J. A. Godoy, and J. L. García-Castaño. 2007. Differential contribution of frugivores to complex seed dispersal patterns. Proceedings of the National Academy of Sciences USA 104:3278-3282.

Lloret, F., J. Peñuelas, and M. Estiarte. 2005. Effects of vegetation canopy and climate on seedling establishment in Mediterranean shrubland. Journal of Vegetation Science 16: 67-76.

Loiselle, B. A., P. G. Blendinger, J. G. Blake, and T. B. Ryder. 2007. Ecological redundancy in seed dispersal systems: a comparison between Manakins (Aves: Pipridae) in two tropical forests. Pages 178-196 in A. J. Dennis, E. W. Schupp, R. J. Green, and D. A. Westcott, editors. Seed dispersal: theory and its application in a changing world. $\mathrm{CAB}$ International, Wallingford, UK.

Manly, B. F. J. 1998. Randomization, bootstrap, and Monte Carlo methods in biology. Chapman and Hall, London, UK. Molina-Borja, M. 1985. Spatial and temporal behaviour of Gallotia galloti in a natural population of Tenerife. Bonner Zoologische Beiträge 36:541-552.

Nagy, K. A., I. A. Girard, and T. K. Brown. 1999. Energetics of free-ranging mammals, reptiles, and birds. Annual Review of Nutrition 19:247-277.

Nathan, R. 2006. Long-distance dispersal of plants. Science 313:786-788.

Olesen, J. M., and A. Valido. 2003. Lizards as pollinators and seed dispersers: an island phenomenon. Trends in Ecology and Evolution 18:177-181.

Pimm, S. L., M. P. Moulton, and L. J. Justice. 1995. Bird extinction in the Central Pacific. Pages 75-87 in J. H. Lawton and R. M. May, editors. Extinction rates. Oxford University Press, Oxford, UK.

Rodríguez, A., M. Nogales, and C. Nieves. 2007. Germination responses of Rubia fruticosa Ait. seed dispersal system in different experimental seasons. African Journal of Ecology 45:361-364.

Rodríguez, A., M. Nogales, B. Rumeu, and B. Rodríguez. 2008. Temporal and spatial variation in the diet of the endemic lizard Gallotia galloti in an insular Mediterranean scrubland. Journal of Herpetology 42:213-222.

Rodríguez-Pérez, J., and A. Traveset. 2010. Seed dispersal effectiveness in a plant-lizard interaction and its consequences for plant regeneration after disperser loss. Plant Ecology 207:269-280

Schupp, E. W., P. Jordano, and J. M. Gómez. 2010. Seed dispersal effectiveness revisited: a conceptual review. New Phytologist 188:333-353.

Sekercioglu, C. H., G. C. Daily, and P. R. Ehrlich. 2004. Ecosystem consequences of bird declines. Proceedings of the National Academy of Sciences USA 101:18042-18047.

Sokal, R. R., and F. J. Rohlf. 1995. Biometry. Third edition. Freeman, New York, New York, USA.

Tylianakis, J. M., R. K. Didham, J. Bascompte, and D. A. Wardle. 2008. Global change and species interactions in terrestrial ecosystems. Ecology Letters 11:1351-1363.

Valido, A. 1999. Ecología de la dispersión de semillas por los lagartos endémicos Canarios (g. Gallotia, Lacertidae). Dissertation. University of La Laguna, Tenerife, Spain.

Vázquez, D. P., W. F. Morris, and P. Jordano. 2005. Interaction frequency as a surrogate for the total effect of animal mutualists on plants. Ecology Letters 8:1088-1094.

Zamora, R. 2000. Functional equivalence in plant-animal interactions: ecological and evolutionary consequences. Oikos 88:442-447.

\section{SuPplemental Material}

\section{Ecological Archives}

Appendices A-C are available online: http://dx.doi.org/10.1890/14-0655.1.sm 